\title{
Physicochemical and Biological Characterization of a Biosimilar Trastuzumab
}

\author{
Carlos A. López-Morales, ${ }^{1}$ Mariana P. Miranda-Hernández, ${ }^{1}$ \\ L. Carmina Juárez-Bayardo, ${ }^{1}$ Nancy D. Ramírez-Ibáñez, ${ }^{1}$ Alexis J. Romero-Díaz, ${ }^{1}$ \\ Nelly Piña-Lara, ${ }^{1}$ Víctor R. Campos-García, ${ }^{2}$ Néstor O. Pérez, ${ }^{1}$ \\ Luis F. Flores-Ortiz, ${ }^{1}$ and Emilio Medina-Rivero ${ }^{1}$ \\ ${ }^{1}$ Unidad de Investigación y Desarrollo, Probiomed S.A. de C.V., Cruce de Carreteras Acatzingo-Zumpahuacán s/n, \\ 52400 Tenancingo, MEX, Mexico \\ ${ }^{2}$ MUHC Research Institute, Royal Victoria Hospital, McGill University, 687 Pine Avenue West, Montreal, QC, Canada H3A 1A1
}

Correspondence should be addressed to Luis F. Flores-Ortiz; luis.flores@probiomed.com.mx and Emilio Medina-Rivero; emilio.medina@probiomed.com.mx

Received 11 October 2014; Revised 10 March 2015; Accepted 24 March 2015

Academic Editor: Pedro H. Oliveira

Copyright (C) 2015 Carlos A. López-Morales et al. This is an open access article distributed under the Creative Commons Attribution License, which permits unrestricted use, distribution, and reproduction in any medium, provided the original work is properly cited.

\begin{abstract}
According to the World Health Organization, the incidence of malignant neoplasms and endocrine, blood, and immune disorders will increase in the upcoming decades along with the demand of affordable treatments. In response to this need, the development of biosimilar drugs is increasing worldwide. The approval of biosimilars relies on the compliance with international guidelines, starting with the demonstration of similarity in their physicochemical and functional properties against the reference product. Subsequent clinical studies are performed to demonstrate similar pharmacological behavior and to diminish the uncertainty related to their safety and efficacy. Herein we present a comparability exercise between a biosimilar trastuzumab and its reference product, by using a hierarchical strategy with an orthogonal approach, to assess the physicochemical and biological attributes with potential impact on its pharmacokinetics, pharmacodynamics, and immunogenicity. Our results showed that the high degree of similarity in the physicochemical attributes of the biosimilar trastuzumab with respect to the reference product resulted in comparable biological activity, demonstrating that a controlled process is able to provide consistently the expected product. These results also constitute the basis for the design of subsequent delimited pharmacological studies, as they diminish the uncertainty of exhibiting different profiles.
\end{abstract}

\section{Introduction}

Biopharmaceutical products containing chimeric, humanized, or fully human monoclonal antibodies (mAbs) are among the most successful and demanded therapies due to their highly specific mechanisms of action that result in an improvement of the patients' conditions and an increase in the survival rate, while minimizing the adverse side-effects when compared to other treatments [1]. Consequently, new manufacturing sites, process scale-ups as well as process improvements contribute to the well-known heterogeneity, naturally present in biotherapeutic products. For this purpose, the ICH Q5 E guideline provides the principles for assessing comparability of licensed biotechnological products subject to process changes throughout their life cycle [2].

In this sense, the approval of biosimilar products, which have been recognized not only as an alternative but as a necessity to increase health coverage and improve the quality of life of patients, follows a similar comparability scheme. International guidelines on biosimilarity [3-5] outline that the approval of biosimilars must rely on the demonstration of comparability towards the reference product, starting with an exhaustive physicochemical and biological characterization whose results will provide evidence to support the extent of additional clinical evaluation [6-8]. 
For this purpose, the proper identification of critical quality attributes (CQAs) that may impact on the pharmacokinetics, pharmacodynamics, and immunogenicity can be achieved through a deep knowledge of the chemical composition and the higher order structure of the active pharmaceutical ingredient (API) contained in the reference product, as well as the known relationships between specific attributes and biological functionality, anticipated by the biotechnological industry and the scientific community [917]. Furthermore, the ICH Q9 guideline highlights the need of evaluating the quality of a biopharmaceutical product based on a risk analysis that considers relevant attributes to the drug's safety and efficacy [18].

In this work we present a comparability study between a biosimilar trastuzumab and its reference product. Trastuzumab is a humanized monoclonal antibody targeted against the extracellular portion of the human epidermal growth factor receptor (HER2, p185), which is overexpressed in approximately 15 to $30 \%$ of the invasive breast cancer cases [19-22]. The chemical, physical, and functional properties closely related to its pharmacological behavior were identified through a risk analysis; then those CQAs were evaluated using several analytical techniques in an orthogonal approach that increases the reliability of the results obtained.

\section{Materials and Methods}

2.1. Materials. Biosimilar trastuzumab ( $440 \mathrm{mg}$ powder for concentrate for solution for infusion) from Probiomed S.A. de C.V. (Mexico City, Mexico) and Herceptin (440 mg powder for concentrate for solution for infusion) from F. Hoffmann, La Roche Ltd. (Basel, Switzerland), were used for the comparability study.

\subsection{Methods}

2.2.1. Physicochemical Properties. Primary sequences, verified from the whole-molecule exact masses and tryptic peptide mappings, were analyzed by reverse phase ultraperformance-liquid-chromatography coupled to a tandem quadrupole/time-of-flight mass spectrometer (RP-UPLCMS/MS). Higher order structure was evaluated by differential scanning calorimetry (DSC), circular dichroism (CD), and fluorescence lifetime using the time correlated single photon counting technique (TCSPC). Charge heterogeneity of the whole, carboxypeptidase-digested, and papaindigested molecule was assessed either by capillary isoelectrofocusing (cIEF) or by cation exchange ultra-performanceliquid-chromatography (CEX-UPLC). Purity was determined by capillary gel electrophoresis under reducing (CGE$\mathrm{R})$ and nonreducing (CGE-NR) conditions and size exclusion ultra-performance-liquid-chromatography (SE-UPLC). Sample treatment and analysis conditions were performed as previously described for RP-UPLC-MS/MS, DSC, CD, CEX, CGE-R, and CGE-NR by Flores-Ortiz et al., 2014 [23]; TCSPC by Pérez Medina Martínez et al., 2014 [24]; cIEF by Espinosa-de la Garza et al. [25].
$\mathrm{N}$-linked glycans were released from trastuzumab by enzymatic hydrolysis using PNGase F from New England Biolabs Inc. (Ipswich, MA) and then were labeled with 8aminopyrene-1,3,6-trisulfonic acid (APTS) and analyzed by capillary zone electrophoresis (CZE) [26]. The electrophoretic separation was carried out in a PA 800 plus Analysis System from Beckman Coulter Inc. (Brea, CA) using an amine coated capillary of $50 \mu \mathrm{m}$ I.D. $\times 50.2 \mathrm{~cm}$ total length, with $40 \mathrm{~cm}$ effective length at $20^{\circ} \mathrm{C}$. Laser induced fluorescence (LIF) detection was used at an excitation wavelength of $488 \mathrm{~nm}$ and emission band-pass filter of $520 \mathrm{~nm}$. An orthogonal analysis was performed by hydrophilic interaction ultraperformance-liquid-chromatography (HILI-UPLC) after labeling with 2-aminobenzoic acid (2-AB) following a previously reported methodology [27].

\subsection{Functional Properties}

2.3.1. Fc $\gamma$ RIIIa Affinity by Isothermal Titration Calorimetry (ITC). Affinity constants under equilibrium $\left(K_{a}\right)$ were obtained from a Nano ITC instrument (TA Instruments Inc.; New Castle, DE). $300 \mu \mathrm{L}$ of Fc $\gamma$ RIIIa solutions at $5.0 \mu \mathrm{M}$ in $\mathrm{PBS}$ at $\mathrm{pH} 7.2$ was titrated with continuous injections of $1.9 \mu \mathrm{L}$ trastuzumab solutions at $50 \mu \mathrm{M}$ in $\mathrm{PBS}$ at $\mathrm{pH}$ 7.2 until saturation at $25^{\circ} \mathrm{C}$. NanoAnalyze Software v2.4.1 (TA Instruments Inc.; New Castle, DE) was used for the integration of heat signals and nonlinear regression analysis of the data.

2.3.2. FcRn Affinity by BLI. Binding kinetics of trastuzumab to FcRn were determined using a Bio-Layer Interferometry (BLI) instrument, Octet QK384, from Pall ForteBio Corp. (Menlo Park, California). Biotinylated FcRn was immobilized to biosensors coated with streptavidin. Binding profiles were displayed by sensograms. Global kinetic analyses were determined using a 2:1 heterogeneous ligand model fit using Rlinked analysis.

2.3.3. HER2 Affinity Assay. HER2 expressing cells SK-BR-3 (ATCC HTB-30) were incubated in the presence of different concentrations of trastuzumab in McCoy-5A medium with $10 \% \mathrm{FBS}$ for $2 \mathrm{~h}$ at $37^{\circ} \mathrm{C}$. HRP-conjugated goat anti-human IgG was added to detect the trastuzumab-SK-BR-3 complex after $1 \mathrm{~h}$ of incubation at $37^{\circ} \mathrm{C}$, using $\mathrm{TMB}$ as substrate for $30 \mathrm{~min}$ at room temperature. Absorption was measured at $450 \mathrm{~nm}$. Test results were expressed as the relative percentage of the $\mathrm{EC}_{50}$ from the dose-response curve of the biosimilar trastuzumab with respect to the reference product.

2.3.4. Antiproliferation Assay. BT-474 cells (ATCC HTB-20) were seeded in DMEM media with $10 \%$ FBS, $1 \%$ nonessential amino acids, and incubated at $37^{\circ} \mathrm{C}$. Different concentrations of trastuzumab were added with further incubation for 8 days. Crystal violet was added to stain the cells for $15 \mathrm{~min}$ at room temperature followed by fixation with formaldehyde and water rising. Acetic acid aqueous solution (33\% v/v) was added to remove the dye excess; absorbance was measured at $540 \mathrm{~nm}$. Test results were expressed as the relative percentage 


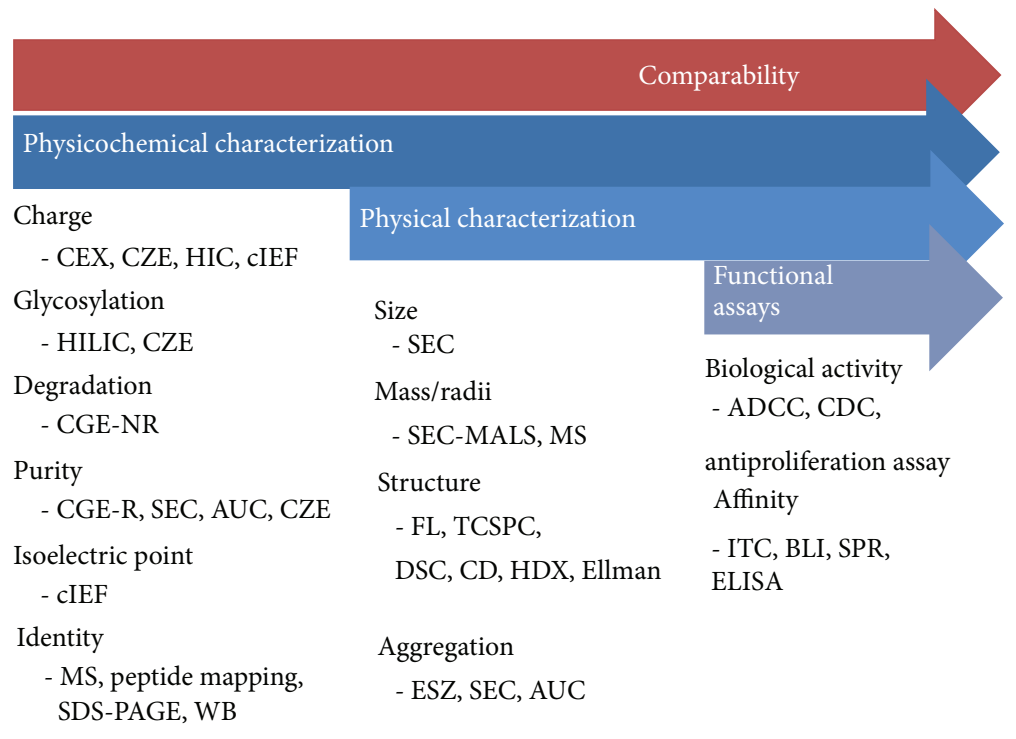

FIgURE 1: Characterization strategy performed for Trastuzumab-Probiomed.

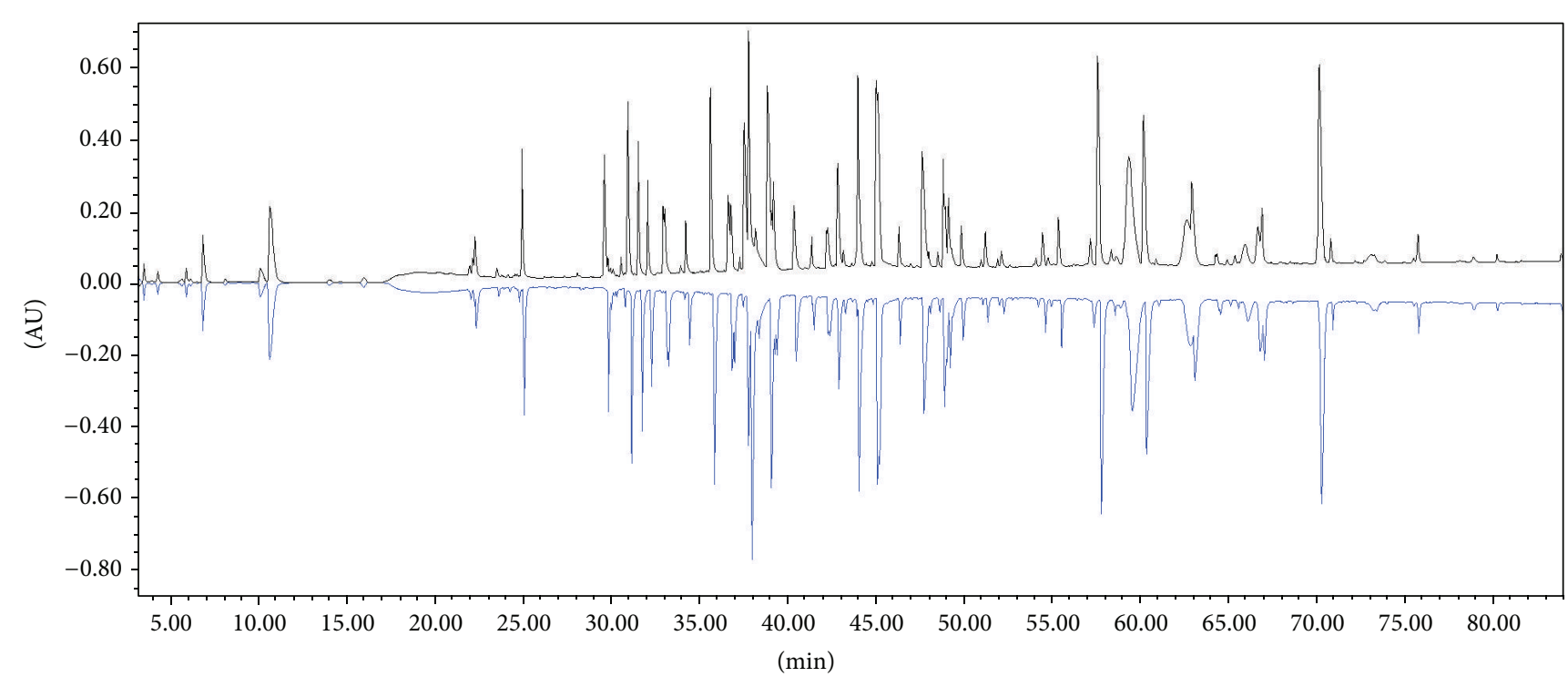

FIGURE 2: Mirror plot of peptide mapping chromatograms obtained from RP-UPLC-UV for Trastuzumab-Probiomed (upper) and the reference product (lower).

of the $\mathrm{EC}_{50}$ from the dose-response curve of the biosimilar trastuzumab with respect to the reference product.

\section{Results and Discussion}

Our characterization strategy (Figure 1) comprised a set of state-of-the-art analytical techniques planned for a hierarchical study of a biosimilar trastuzumab using an orthogonal approach. CQAs were identified using a risk analysis, considering each of the physicochemical and functional properties that may have an impact on efficacy (pharmacokinetics and pharmacodynamics) and safety (immunogenicity) of trastuzumab (Table 1) [9-17]. In this work, only certain methodologies were selected to depict a global overview of the characterization study. Hereafter, CQAs were classified by their physicochemical, physical, or biological nature and analyzed comparatively for a biosimilar trastuzumab (Trastuzumab-Probiomed) and its reference product.

3.1. Physicochemical Properties. The identity of TrastuzumabProbiomed towards the reference product was determined by the correspondence of their tryptic peptide mappings (Figure 2). MS/MS analysis verified the amino acid sequence of both products against the theoretical stated on the invention patent of trastuzumab [28], unveiling a sequence matching of $99.8 \%$ and $99.3 \%$ for the heavy chain and $99.5 \%$ and 99.5\% for the light chain, for both Trastuzumab-Probiomed and the reference product, respectively (Figures 3 and 4). 


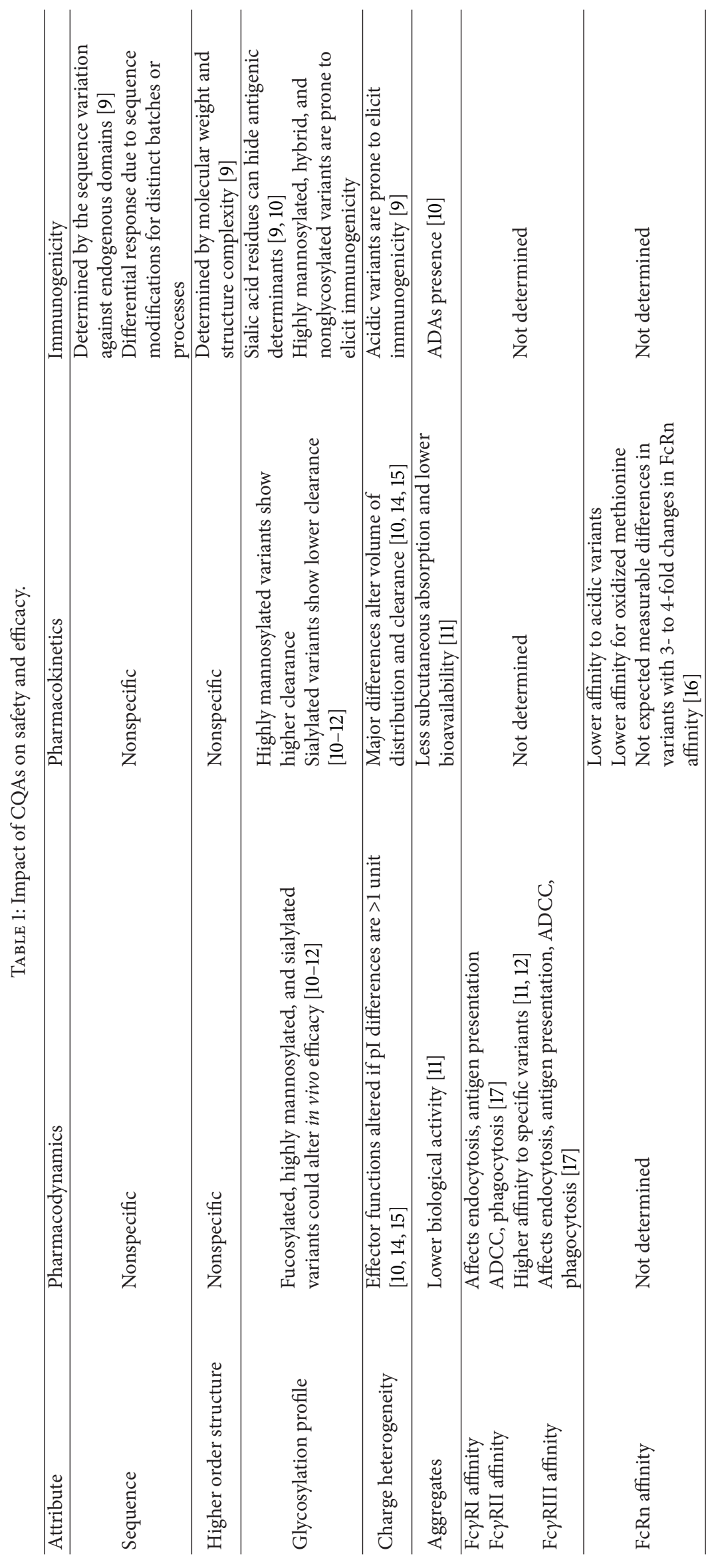


LC

Control coverage (\%): 99.5 Control unique coverage (\%): 99.5

$\begin{array}{ll}1: 1 \text { to } 50 & \text { D I Q T Q S P S } \\ 1: 51 \text { to } 100 & \text { A S F L Y S V P S } \\ 1: 101 \text { to } 150 & \text { G T K V E I K T V } \\ 1: 151 \text { to } 200 & \text { D N A L S G N Q } \\ 1: 201 \text { to } 214 & \text { L S S P V T K F N }\end{array}$

Control coverage (\%): 99.8

Control unique coverage (\%): 99.8

\begin{tabular}{|c|c|}
\hline $1: 1$ to 50 & E V Q L V E S G G G \\
\hline $1: 51$ to 100 & I Y P T N G Y T R Y \\
\hline $1: 101$ to 150 & G D G F Y A M D Y W \\
\hline $1: 151$ to 200 & D Y F P E P V T V S \\
\hline $1: 201$ to 250 & Y I C N V N H K P S \\
\hline $1: 251$ to 300 & K D T L M I S R P P \\
\hline $1: 301$ to 350 & S T Y R V V S V L T \\
\hline $1: 351$ to 400 & VYT L P P SREE \\
\hline $1: 401$ to 450 & LDSDGSFF L Y \\
\hline
\end{tabular}

Combined coverage (\%): 99.5

Common coverage (\%): 0.0

$\begin{array}{ll}\text { LSASVGDRT } & \text { ITCRASQDVN } \\ \text { RFSGSRGTD } & \text { FTLTISSLQP } \\ \text { AAPSVFIFPP } & \text { SDEQLKSGTA } \\ \text { ESVTEQDSKD } & \text { STYSLSSTLT } \\ \text { RGEC } & \end{array}$

$\mathrm{HC}$

Combined coverage (\%): 99.8

Common coverage (\%): 0.0

LVQPGGSLRL
ADSVKGRTI
GQGTLVTVSS
WNSGALT GV
N TKVDKKVEP
EVTCVVVDVS
VLHQDWLNGK
MTKNQVSLTC
SKLTVDKSRW

\begin{tabular}{|c|c|c|}
\hline C A A S G F N I K & D T Y I H W V R Q A & P G K G L E W V \\
\hline A D T S K N T A Y & L Q M N S L R A E D & T A V Y Y C S R W G \\
\hline A S T K G P S V F P & L A P S SK S T S G & G T A A L G C L V K \\
\hline H T F P A V L Q S S & G L Y S L S S V V T & VPSS L L T Q T \\
\hline K S C D K T H T C P & P C P A P E L L G G & P S V F L F P P K P \\
\hline H E D P E V K F N W & Y V D G V E V H N A & K T K P R E E Q Y N \\
\hline E Y K C K V S N K A & L P A P I E K T I S & KAKGQPR EPQ \\
\hline L V K G F Y P S D I & A V E W E S N GQP & E N N Y K T T P P V \\
\hline QQ G & M H E A L H N H Y T & Q K S L \\
\hline
\end{tabular}

Analyte coverage (\%): 0.0

Analyte unique coverage (\%): 0.0

FIGURE 3: Sequence coverage of the heavy and light chains of Trastuzumab-Probiomed obtained from the MS/MS analysis.

This correspondence was further confirmed by the analyses of the exact masses against the theoretical mass [28, 29] for both whole and deglycosylated molecules (Tables 2 and 3 ). The sequences coverage confirms that the amino acid sequence of Trastuzumab-Probiomed is identical to the reference product, while the $<25 \mathrm{Da}$ observed differences in intact masses for the whole molecule, below the expected width of the isotopic pattern distribution of a mAb, show in advance a comparable degree of heterogeneity, due to posttranslational modifications, in both products, ultimately producing an equivalent immunogenic response.

Regarding glycan microheterogeneity, which is known to contribute to the correct folding and stability of a mAb, it was analyzed by CZE and HILI-UPLC. Particularly, highly mannosylated and sialylated glycoforms are reported to alter a mAb half-life in blood and are linked to potential immunogenic responses; moreover effector functions can be altered due to the presence of highly mannosylated, bisected, and fucosylated glycoforms, as a consequence of charge or steric hindrances [10-12].

CZE analyses revealed that the glycan patterns of Trastuzumab-Probiomed and the reference product are comprised of the same principal glycoforms (Figure 5(a)), showing a mean relative abundance of galactosylated variants of $66.01 \%$ and $49.57 \% \pm 6.18$ (CI 95\%) for TrastuzumabProbiomed and the reference product, respectively, which is not expected to have an impact on the functional properties, since galactosylation has not been reported to alter the mechanisms of action of mAbs, as confirmed by the affinities and biological potency analyses discussed below. Further analysis by HILI-UPLC of the glycoforms identified as critical for PK, PD, or immunogenicity (Table 1) revealed comparable relative abundances of highly mannosylated variants, being $2.00 \pm 0.10$ (CI 95\%) and $3.96 \pm 0.45$ (CI 95\%) for Trastuzumab-Probiomed and the reference product, respectively, whereas the mean abundance for hybrid and sialylated variants was $4.75 \pm 0.19$ (CI 95\%) and $0.27 \pm$ 0.08 (CI 95\%) for the reference product and $2.95 \pm 0.15$ (CI 95\%) and $1.06 \pm 0.14$ (CI 95\%) for Trastuzumab-Probiomed, respectively. These results confirm similarity of the critical glycoforms between Trastuzumab-Probiomed and the reference product; thus similar PK and PD profiles and no differential immunogenicity response are expected.

On the other hand, charge heterogeneity evaluated through cIEF analysis revealed that isoelectric points (pI) for the main isoform were $8.69 \pm 0.00$ (CI 95\%) for TrastuzumabProbiomed and $8.70 \pm 0.01(\mathrm{CI} 95 \%)$ for the reference product, in accordance with the expected $\mathrm{pI}$ variations during manufacturing, no larger than 0.2 units $[15,16]$. The observed isoform-abundance-weighted $\mathrm{pI}$ values confirmed similarity of charge heterogeneity among products, being $8.60 \pm 0.01$ (CI 95\%) for Trastuzumab-Probiomed and $8.61 \pm 0.01$ (CI $95 \%)$ for the reference product. It has been reported that only changes in one $\mathrm{pI}$ unit can significantly alter the therapeutic activity of a $\mathrm{mAb}$; thus the observed variation is not expected to affect the clinical behavior of Trastuzumab-Probiomed with respect to the reference product.

An orthogonal analytical technique for the evaluation of charge heterogeneity was CEX-UPLC, which revealed that the averaged abundances of the main, acidic, and basic 
Control coverage (\%): 99.5

Control unique coverage (\%): 99.5

\section{$1: 1$ to 50}

$1: 51$ to 100

$1: 101$ to 150

$1: 151$ to 200

$1: 201$ to 214

Control coverage (\%): 99.3

Control unique coverage (\%): 99.3

\section{$1: 1$ to 50 EVQLVESGG G \\ $1: 51$ to 100 I Y P T N G Y T R Y \\ $1: 101$ to 150 G D GF Y A M D Y W \\ $1: 151$ to 200 D Y F P E P V T V S \\ $1: 201$ to 250 Y I C N V N H K P S \\ $1: 251$ to 300 K D T L M I S R T P \\ $1: 301$ to $350 \quad$ S T Y R V V S V L T \\ $1: 351$ to 400 V Y T L P P SREE \\ $1: 401$ to 450 L D S D G S F F L Y}

LC

Combined coverage (\%): 99.5

Common coverage (\%): 0.0

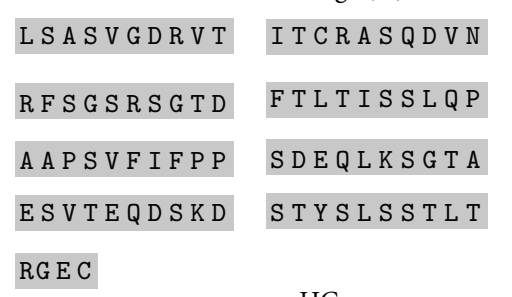

$\mathrm{HC}$

Combined coverage (\%): 99.3

Common coverage (\%): 0.0

\section{V QPGGSLRL \\ A D S V K GRF T I \\ GQG T L V T V S S \\ W N S G A L T S G V \\ N T K V D K K V E P \\ E V T C V V V D V S \\ V L H Q D W L N G K \\ M T K N Q V S L T C \\ S K L T V D K S R W}

\section{S C A A S G F N I K \\ S A D T S K N T A Y \\ A S T K G P S V F P \\ H T F P A V L Q S S \\ K S C D K T H T C P \\ H E D P E V K F N W \\ E Y K C K V S N K A \\ L V K G F Y P S D I \\ QQGN V F S C S V}

Analyte coverage (\%): 0.0 Analyte unique coverage (\%): 0.0

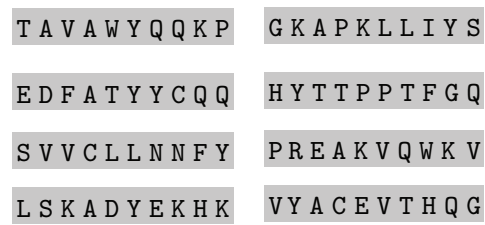

Analyte coverage (\%): 0.0 Analyte unique coverage (\%): 0.0

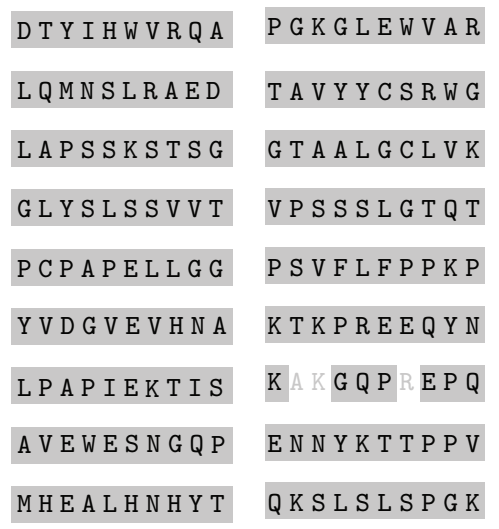

FIGURE 4: Sequence coverage of the heavy and light chains of the reference product obtained from the MS/MS analysis.

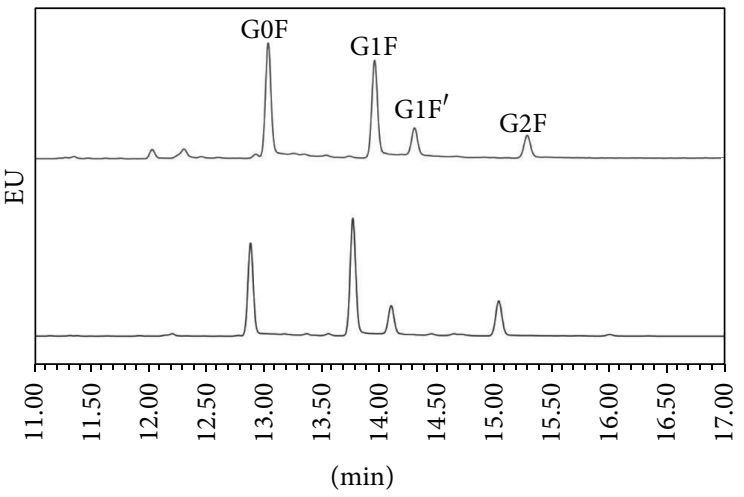

(a)

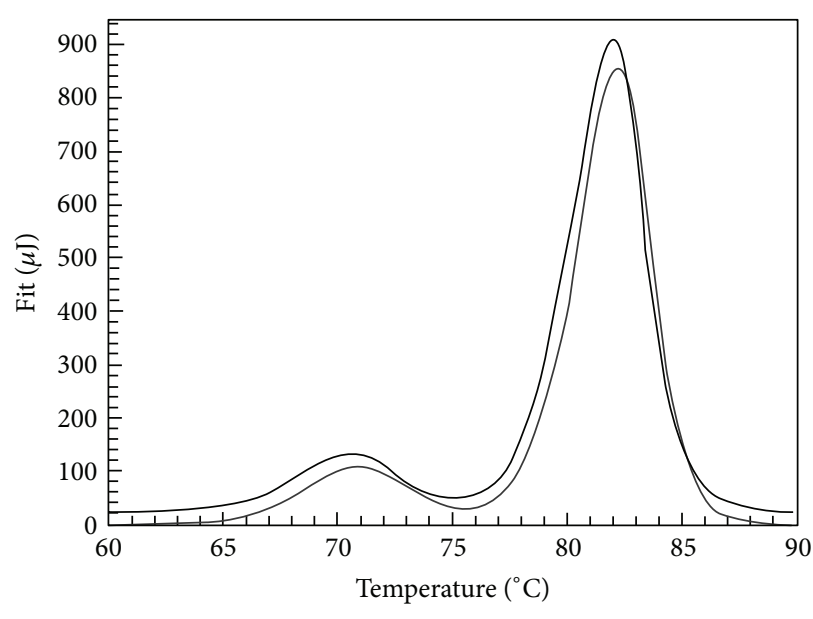

(b)

FIGURE 5: (a) Glycan profile for the reference product (upper) and Trastuzumab-Probiomed (lower). (b) Thermostability by DSC for the reference product (lower) and Trastuzumab-Probiomed (upper).

isoforms were within the same order of magnitude for both products, being the mean values of $57.0 \%, 33.2 \%$, and $9.8 \%$ $(n=3)$ for Trastuzumab-Probiomed and 62.5\%, 27.3\%, and $10.3 \%(n=3)$ for the reference product, respectively. Furthermore, the results obtained after digestion with carboxypeptidase B showed also a comparable content of basic, acidic, and main isoforms among the two products, with a main relative content of $16.4 \%, 30.6 \%$, and $53.0 \%(n=3)$ for the reference product and $8.8 \%, 37.8 \%$, and $53.4 \%(n=3)$ for Trastuzumab-Probiomed, respectively.

After papain digestion, the mean abundance of basic isoforms in the reference product $(n=3)$ was $3.8 \%$ for 
TABLE 2: Whole-molecule exact masses by MS.

\begin{tabular}{lccccccc}
\hline Product & Batch & G0/G0F & G0F/G0F & G0F/G1F & G1F/G1F & G1F/G2F & G2F/G2F \\
\hline Averaged theoretical & - & 147911.76 & 148057.91 & 148220.05 & 148382.19 & 148544.33 & 148706.46 \\
& B3417B010 & 147907.81 & 148061.92 & 148220.20 & 148378.84 & 148536.86 & 148692.48 \\
Reference product & B3433B010 & 147897.68 & 148058.03 & 148218.21 & 148377.11 & 148534.93 & 148690.07 \\
& N3477B021 & 147899.82 & 148058.00 & 148218.10 & 148377.32 & 148535.39 & 148690.95 \\
\hline \multirow{3}{*}{ Trastuzumab-Probiomed } & TZPP12001 & 147901.19 & 148057.88 & 148218.18 & 148378.54 & 148537.97 & 148695.60 \\
& TZPP12002 & 147900.45 & 148057.89 & 148217.99 & 148378.23 & 148537.59 & 148694.92 \\
& TZPP12003 & 147898.55 & 148057.58 & 148217.49 & 148377.84 & 148537.14 & 148694.42 \\
\hline
\end{tabular}

TABLE 3: Deglycosylated molecule exact masses by MS.

\begin{tabular}{lcc}
\hline Product & Batch & Mass (Da) \\
\hline Theoretical & - & 145167.36 \\
\hline \multirow{3}{*}{ Reference product } & B3417B010 & 145167.47 \\
& B3433B010 & 145167.36 \\
& N3477B021 & 145167.16 \\
\hline \multirow{3}{*}{ Trastuzumab-Probiomed } & TZPP12002 & 145167.53 \\
& TZPP12001 & 145167.08 \\
& TZPP12003 & 145167.69 \\
\hline
\end{tabular}

the Fc fragment and $4.9 \%$ for the Fab fragment, whereas for Trastuzumab-Probiomed $(n=3)$ it was $4.2 \%$ for the Fc fragment and $6.5 \%$ for the Fab fragment. Regarding acidic isoforms, the mean abundance was $3.3 \%$ for the $\mathrm{Fc}$ fragment and $16.7 \%$ for the Fab fragment of the reference product, while for Trastuzumab-Probiomed it was 3.7\% for the Fc fragment and $16.1 \%$ for the Fab fragment. Finally, the abundance of the $\mathrm{Fc}$ and $\mathrm{Fab}$ fragments was $26.7 \%$ and $44.6 \%$, respectively, for the reference product, and for TrastuzumabProbiomed the abundance of the Fc and Fab fragments was $25.7 \%$ and $43.8 \%$, respectively.

Overall the results from CIEF and CEX-UPLC show that both products exhibit comparable charge heterogeneities, either as a whole molecule or as the fragments responsible for the recognition and effector functions of trastuzumab; thus no differences in functional activity should be expected.

CGE-NR and SE-UPLC results demonstrated that both products have a similar degree of purity (Tables 4 and 5) based on the relative content of monomer with respect to the presence of aggregates and other degraded or truncated isoforms. It is known that protein aggregation can induce immunogenicity; although a small amount of aggregates is expected, this amount is likely to increase due to stress conditions that a $\mathrm{mAb}$ may undergo during its manufacture, purification, formulation, and shelf-life $[9,30]$. Aggregation may reveal new epitopes that potentially could stimulate the production of anti-drug antibodies (ADAs) resulting in the loss of activity, immunogenic reactions, or adverse effects during administration. Likewise, the presence of fragments or truncated forms coming from hydrolysis reactions could negatively impact on the safety and therapeutic effect of a $\mathrm{mAb}[31,32]$. The content of aggregates and truncated forms
TABLE 4: Monomer content of trastuzumab by SE-UPLC and CGENR. Variation is presented as confidence intervals at $95 \%(n=3)$.

\begin{tabular}{lccc}
\hline Product & Batch & SE-UPLC (\%) & CGE-NR (\%) \\
\hline Trastuzumab- & TZPP11002 & $99.6 \pm 0.0$ & $92.3 \pm 0.3$ \\
Probiomed & TZPP12001 & $98.9 \pm 0.0$ & $90.8 \pm 1.1$ \\
& TZPP11001 & $99.4 \pm 0.0$ & $96.6 \pm 0.4$ \\
\hline \multirow{3}{*}{ Reference product } & N3597B013 & $98.9 \pm 0.1$ & $92.8 \pm 0.6$ \\
& N35973 & $99.7 \pm 0.0$ & $93.5 \pm 0.7$ \\
& B34310 & $99.5 \pm 0.0$ & $93.1 \pm 0.4$ \\
\hline
\end{tabular}

of Trastuzumab-Probiomed were lower than the limits established by the USP [29] and were comparable to the reference product; thus the risk of developing a different immunogenic response (differential immunogenicity) is diminished.

3.2. Physical Properties. Since the functionality of trastuzumab is affected by its three-dimensional structure, which results from its primary sequence and posttranslational modifications that alter its size, mass, folding, and stability [8], we performed analyses to assess the spatial configuration of Trastuzumab-Probiomed compared to its reference product. Time correlated single photon counting analysis (TCSPC) was employed to evaluate the fluorescence lifetime $(\tau)$, which depends on the exposure of aromatic amino acids within the protein, thus demonstrating similarity when the results are obtained from comparative analyses [33-36]. TCSPC results showed that the averaged $\tau$ of Trastuzumab-Probiomed was $3.43 E^{-09} \pm 1.39 E^{-10} \mathrm{~s}$ (CI 95\%), while the averaged $\tau$ for the reference product was $3.49 E^{-09} \pm 1.69 E^{-11}$ s (CI 95\%). Regarding $\mathrm{CD}$, the obtained spectrograms were superimposable in both near- and far-UV regions (Figure 6) suggesting that alpha helix, beta sheets, random coil, disulfide bonds, and aromatic amino acids are distributed in a comparable spatial arrangement. Finally, transition temperatures $\left(T_{m}\right)$ of Trastuzumab-Probiomed $(n=3)$ by DSC (Figure 5(b)) were $70.4^{\circ} \mathrm{C}, 79.1^{\circ} \mathrm{C}, 81.0^{\circ} \mathrm{C}$, and $82.5^{\circ} \mathrm{C}$, whereas for the reference product $(n=3)$ they were $70.5^{\circ} \mathrm{C}, 79.6^{\circ} \mathrm{C}, 81.2^{\circ} \mathrm{C}$, and $82.7^{\circ} \mathrm{C}$; for both products the $\mathrm{CI}$ at $95 \%$ was $<0.02^{\circ} \mathrm{C}$ for all the temperatures. Collectively TCSPC, CD, and DSC determined that thermostability and secondary and tertiary structures of Trastuzumab-Probiomed were comparable to the reference product. In particular, thermostability results are indicative of a proper protein folding of both products in their respective 
TABLE 5: Relative abundance of trastuzumab subunits by CGE-R. Variation is presented as confidence interval at $95 \%(n=3)$.

\begin{tabular}{lcccr}
\hline Product & Batch & HC \% & NGHC \% & LC \% \\
\hline \multirow{3}{*}{ Trastuzumab-Probiomed } & TZPP12001 & $66.18 \pm 0.16$ & $0.57 \pm 0.06$ & $32.89 \pm 0.19$ \\
& TZPP12002 & $64.46 \pm 0.47$ & $0.58 \pm 0.02$ & $34.59 \pm 0.32$ \\
& TZPP12003 & $65.45 \pm 0.97$ & $0.50 \pm 0.05$ & $33.53 \pm 1.08$ \\
\hline \multirow{3}{*}{ Reference product } & B3393B019 & $65.14 \pm 0.25$ & $0.57 \pm 0.03$ & $33.93 \pm 0.13$ \\
& B3417B010 & $66.02 \pm 0.27$ & $0.52 \pm 0.09$ & $33.04 \pm 0.25$ \\
& B3430 & $66.40 \pm 0.25$ & $0.63 \pm 0.01$ & $32.46 \pm 0.16$ \\
\hline
\end{tabular}

HC: heavy chain, NGHC: nonglycosylated heavy chain, and LC: light chain.

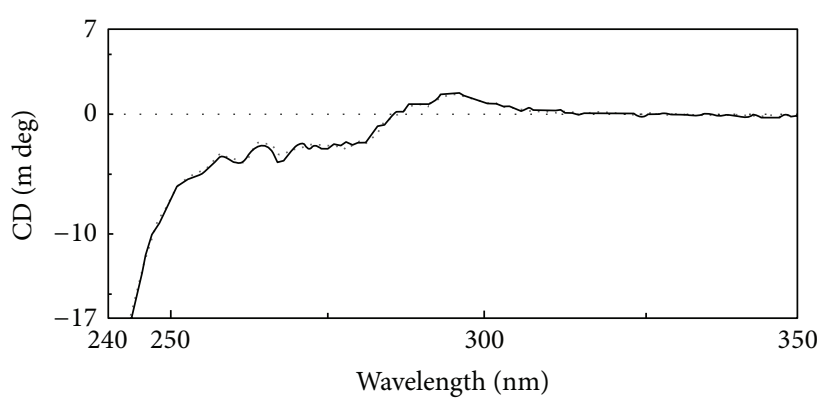

(a)

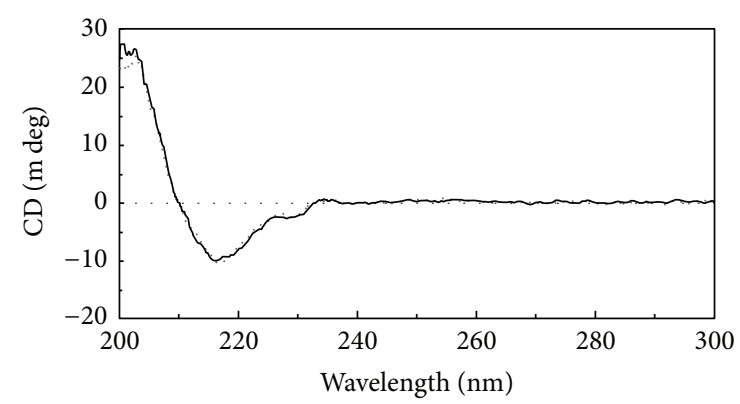

(b)

FIGURE 6: Analysis of the three-dimensional structure of trastuzumab by CD of Trastuzumab-Probiomed (solid line) and the reference product (dotted line) in both near-UV region (a) and far-UV region (b).

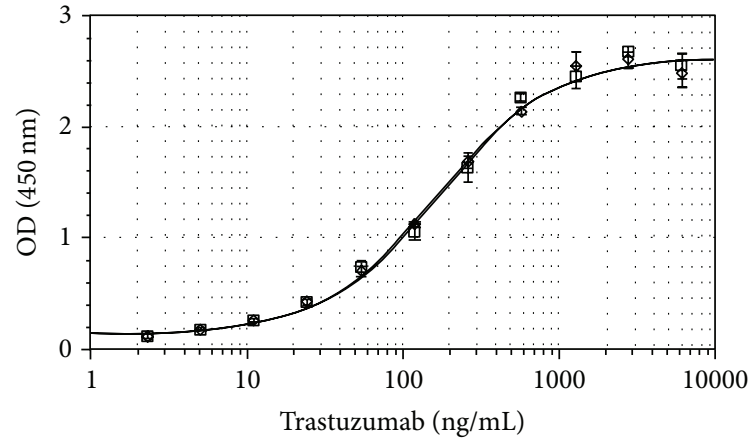

(a)

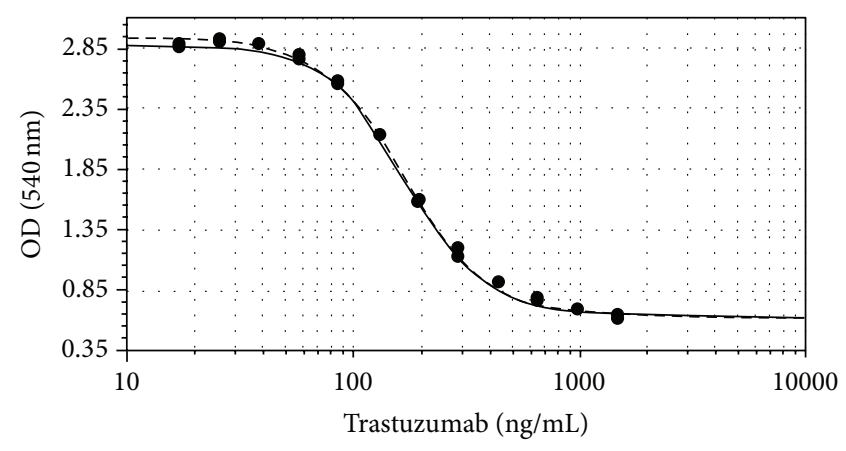

(b)

FIGURE 7: Comparison of in vitro activity between Trastuzumab-Probiomed and the reference product. (a) Curve of binding affinity to HER2; (b) potency curve obtained from the antiproliferation assay; the solid line corresponds to Trastuzumab-Probiomed, while the dashed line corresponds to the reference product.

formulation. This physicochemical and physical similarity is the major contributor to equivalent biological and functional responses.

3.3. Functional Properties. The relative affinity of Trastuzumab-Probiomed towards its target molecule, HER2 (Figure 7(a) and Table 7), was evaluated with respect to the reference product, resulting in an averaged relative affinity of $97.7 \%$. Thus, it is expected that Trastuzumab-Probiomed can exert its activity through the reported mechanisms of action, including HER2 downregulation, prevention of the heterodimer formation, initiation of $\mathrm{Gl}$ arrest, induction of $\mathrm{p} 27$, and prevention of HER2 cleavage [37].
The main mechanisms of action rely on the affinity of the Fc fragment of trastuzumab towards $F c \gamma$ receptors. For instance, Fc $\gamma$ RIIIa present on effector cells such as macrophages, monocytes, and natural killer cells activates and induces ADCC mechanism against HER2-positive cells $[37,38]$. Binding affinities towards Fc $\gamma$ RIIIa were evaluated by ITC, being the averaged affinity constants $\left(K_{a}\right)$ of $2.61 \pm 0.54 E^{+06} \mathrm{M}^{-1}$ for Trastuzumab-Probiomed and $2.48 \pm$ $0.30 E^{+06} \mathrm{M}^{-1}$ for the reference product (Table 6). Likewise, the mean dissociation constants $\left(K_{D}\right)$ to FcRn, which regulates IgG catabolism, were determined by BLI as $2.58 E^{-07} \mathrm{M}$ $\pm 1.02 E^{-07} \mathrm{M}$ (CI 95\%) for Trastuzumab-Probiomed with a relative binding affinity of $114.3 \%(n=3)$ with respect to 
TABLE 6: Affinity of trastuzumab to Fc $\gamma$ RIIIa.

\begin{tabular}{lcc}
\hline Product & Batch & $\begin{array}{c}\text { Affinity constant }\left(K_{a}\right) \text { to } \\
\text { Fc } \gamma \text { RIIIa }\left(\mathrm{M}^{-1}\right)\end{array}$ \\
\hline Trastuzumab- & TZPP14001 & $2.71 E+06$ \\
Probiomed & TZPP12002 & $2.86 E+06$ \\
& TZPP12003 & $2.25 E+06$ \\
\hline \multirow{3}{*}{ Reference product } & $\mathrm{N} 35893$ & $2.66 E+06$ \\
& $\mathrm{~N} 35812$ & $2.48 E+06$ \\
& $\mathrm{~N} 36003$ & $2.31 E+06$ \\
\hline
\end{tabular}

TABLE 7: Binding affinity of trastuzumab to the epidermal growth factor receptor (HER2).

\begin{tabular}{lcc}
\hline Product & Batch & $\begin{array}{c}\text { Relative affinity } \\
(\%)\end{array}$ \\
\hline Trastuzumab- & TZPP11001 & 98 \\
Probiomed & TZPP12004 & 98 \\
& TZPP12003 & 97 \\
\hline \multirow{3}{*}{ Reference product } & N3654 & 119 \\
& N36263 & 111 \\
& N36443 & 112 \\
\hline
\end{tabular}

the reference product. Based on these results no differences in the half-life in blood are expected.

The overall in vitro activity was tested between Trastuzumab-Probiomed and the reference product with an antiproliferation assay (Figure 7(b)), which demonstrated that both products have the same potency to deplete HER2positive cells, being the mean relative potencies towards the reference product of $105 \%, 103 \%$, and $110 \%$ for three different batches of Trastuzumab-Probiomed, demonstrating that similarity on physicochemical and physical critical quality attributes results in a comparable biological potency.

\section{Conclusions}

During the development of a biosimilar, an extended characterization of its physicochemical and functional properties is required to gain a strong knowledge of its CQAs. This allows the establishment of in-process control strategies and quality specifications to ensure batch-to-batch consistency in order to obtain the desired product, despite the fact that it has been produced using a different manufacturing process with respect to the reference product. In addition, the use of orthogonal methods during a comparability study provides a global overview of the molecule and confirms the observed results on relevant modifications. Here, it was demonstrated that similarity between the critical physicochemical attributes resulted in comparable biological properties.

The observed physicochemical and functional similarity between products, as part of the totality-of-the-evidence scheme, will determine the extent of upcoming nonclinical and clinical studies, considering that it diminishes the uncertainty of exhibiting different pharmacological profiles.

\section{Conflict of Interests}

Carlos A. López-Morales, Mariana P. Miranda-Hernández, L. Carmina Juárez-Bayardo, Nancy D. Ramírez-Ibáñez, Alexis J. Romero-Díaz, Nelly Piña-Lara, Néstor O. Pérez, Luis F. Flores-Ortiz, and Emilio Medina-Rivero are employees of Probiomed S.A. de C.V., which is developing, manufacturing, and marketing biosimilar products. Víctor R. Campos-García declared no conflict of interests.

\section{Acknowledgment}

Financial support was provided by the National Council for Science and Technology (CONACYT), Mexico, Grant FINNOVA 174102, without participation in the design of the study.

\section{References}

[1] M. McCamish and G. Woollett, "Worldwide experience with biosimilar development," mAbs, vol. 3, no. 2, pp. 209-217, 2011.

[2] ICH, "ICH Q5E: comparability of Biotechnological/Biological products subject to changes in their manufacturing process," in Proceedings of the International Conference on Harmonisation of Technical Requirements for Registration of Pharmaceuticals for Human Use, November 2004.

[3] Food and Drug Administration, Guidance for Industry: Scientific Considerations in Demonstrating Biosimilarity to a Reference Product (Draft), FDA, Rockville, Md, USA, 2012.

[4] Food and Drug Administration, Guidance for Industry: Quality Considerations in Demonstrating Biosimilarity to a Reference Protein Product, (Draft), FDA, Rockville, Md, USA, 2012.

[5] Food and Drug Administration, Guidance for Industry: Clinical Pharmacology Data to Support a Demostration of Biosimilarity to a Reference Product, draft, FDA, Rockville, Md, USA, 2014.

[6] S. K. Jung, K. H. Lee, J. W. Jeon et al., "Physicochemical characterization of Remsima," $m A b s$, vol. 6, no. 5, pp. 1163-1177, 2014.

[7] A. Beck, H. Diemer, D. Ayoub et al., "Analytical characterization of biosimilar antibodies and Fc-fusion proteins," Trends in Analytical Chemistry, vol. 48, pp. 81-95, 2013.

[8] S. A. Berkowitz, J. R. Engen, J. R. Mazzeo, and G. B. Jones, "Analytical tools for characterizing biopharmaceuticals and the implications for biosimilars," Nature Reviews Drug Discovery, vol. 11, no. 7, pp. 527-540, 2012.

[9] G. Shankar, C. Pendley, and K. E. Stein, "A risk-based bioanalytical strategy for the assessment of antibody immune responses against biological drugs," Nature Biotechnology, vol. 25, no. 5, pp. 555-561, 2007.

[10] W. S. Putnam, S. Prabhu, Y. Zheng, M. Subramanyam, and Y.-M. C. Wang, "Pharmacokinetic, pharmacodynamic and immunogenicity comparability assessment strategies for monoclonal antibodies," Trends in Biotechnology, vol. 28, no. 10, pp. 509-516, 2010.

[11] L. Liu, A. Stadheim, L. Hamuro et al., "Pharmacokinetics of IgG1 monoclonal antibodies produced in humanized Pichia pastoris with specific glycoforms: a comparative study with CHO produced materials," Biologicals, vol. 39, no. 4, pp. 205210, 2011. 
[12] T. T. Junttila, K. Parsons, C. Olsson et al., "Superior in vivo efficacy of afucosylated trastuzumab in the treatment of HER2amplified breast cancer," Cancer Research, vol. 70, no. 11, pp. 4481-4489, 2010.

[13] H. Li and M. d'Anjou, "Pharmacological significance of glycosylation in therapeutic proteins," Current Opinion in Biotechnology, vol. 20, no. 6, pp. 678-684, 2009.

[14] J. Sharifi, L. A. Khawli, J. L. Hornick, and A. L. Epstein, "Improving monoclonal antibody pharmacokinetics via chemical modification," The Quarterly Journal of Nuclear Medicine, vol. 42, no. 4, pp. 242-249, 1998.

[15] T. Igawa, H. Tsunoda, T. Tachibana et al., "Reduced elimination of IgG antibodies by engineering the variable region," Protein Engineering, Design and Selection, vol. 23, no. 5, pp. 385-392, 2010.

[16] L. A. Khawli, S. Goswami, R. Hutchinson et al., "Charge variants in IgG1: isolation, characterization, in vitro binding properties and pharmacokinetics in rats," $m A b s$, vol. 2, no. 6, pp. 613-624, 2010.

[17] J. E. Gessner, H. Heiken, A. Tamm, and R. E. Schmidt, “The IgG Fc receptor family," Annals of Hematology, vol. 76, no. 6, pp. 231248, 1998.

[18] ICH, "ICH Q9: quality risk management," in Proceedings of the International Conference on Harmonisation of Technical Requirements for Registration of Pharmaceuticals for Human Use, 2005.

[19] D. J. Slamon, G. M. Clark, S. G. Wong, W. J. Levin, A. Ullrich, and W. L. McGuire, "Human breast cancer: correlation of relapse and survival with amplification of the HER-2/neu oncogene," Science, vol. 235, no. 4785, pp. 177-182, 1987.

[20] D. J. Slamon, W. Godolphin, L. A. Jones et al., "Studies of the HER-2/neu proto-oncogene in human breast and ovarian cancer," Science, vol. 244, no. 4905, pp. 707-712, 1989.

[21] H. J. Burstein, "The distinctive nature of HER2-positive breast cancers," The New England Journal of Medicine, vol. 353, no. 16, pp. 1652-1654, 2005.

[22] Z. Mitri, T. Constantine, and R. O'Regan, “The HER2 receptor in breast cancer: pathophysiology, clinical use, and new advances in therapy," Chemotherapy Research and Practice, vol. 2012, Article ID 743193, 7 pages, 2012.

[23] L. F. Flores-Ortiz, V. R. Campos-García, F. C. PerdomoAbúndez, N. O. Pérez, and E. Medina-Rivero, "Physicochemical properties of Rituximab," Journal of Liquid Chromatography \& Related Technologies, vol. 37, no. 10, pp. 1438-1452, 2014.

[24] V. Pérez Medina Martínez, M. E. Abad-Javier, A. J. RomeroDíaz et al., "Comparability of a three-dimensional structure in biopharmaceuticals using spectroscopic methods," Journal of Analytical Methods in Chemistry, vol. 2014, Article ID 950598, 11 pages, 2014.

[25] C. E. Espinosa-de la Garza, F. C. Perdomo-Abúndez, J. PadillaCalderón et al., "Analysis of recombinant monoclonal antibodies by capillary zone electrophoresis," Electrophoresis, vol. 34, no. 8, pp. 1133-1140, 2013.

[26] Beckman Coulter, CE Separation of N-Linked Oligosaccharides Released from Recombinant Monoclonal Antibody, Application Information, Beckman Coulter, Pasadena, Calif, USA, 2004.

[27] M. P. Miranda-Hernández, C. A. López-Morales, N. D. Ramírez-Ibáñez et al., "Assessment of physicochemical properties of rituximab related to its immunomodulatory activity," Journal of Immunology Research. In press.

[28] J. P. Carter and L. G. Presta, "Immunoglobulin variants," US Patent 5821337, 1998.
[29] The United States Pharmacopeial Convention, “Trastuzumab," Medicines compendium, Versión: 1.0, 2013.

[30] S. N. Telikepalli, O. S. Kumru, C. Kalonia et al., "Structural characterization of IgG1 mAb aggregates and particles generated under various stress conditions," Journal of Pharmaceutical Sciences, vol. 103, no. 3, pp. 796-809, 2014.

[31] T. Ishikawa, N. Kobayashi, C. Osawa, E. Sawa, and K. Wakamatsu, "Prevention of stirring-induced microparticle formation in monoclonal antibody solutions," Biological and Pharmaceutical Bulletin, vol. 33, no. 6, pp. 1043-1046, 2010.

[32] A. J. Cordoba, B.-J. Shyong, D. Breen, and R. J. Harris, "Non-enzymatic hinge region fragmentation of antibodies in solution," Journal of Chromatography B: Analytical Technologies in the Biomedical and Life Sciences, vol. 818, no. 2, pp. 115-121, 2005.

[33] V. Kayser, N. Chennamsetty, V. Voynov, B. Helk, and B. L. Trout, "Tryptophan-tryptophan energy transfer and classification of tryptophan residues in proteins using a therapeutic monoclonal antibody as a model," Journal of Fluorescence, vol. 21, no. 1, pp. 275-288, 2011.

[34] P. R. Callis, “ ${ }^{1} \mathrm{~L}_{a}$ and ${ }^{1} \mathrm{~L}_{b}$ transitions of tryptophan: applications of theory and experimental observations to fluorescence of proteins," Methods in Enzymology, vol. 278, pp. 113-150, 1997.

[35] R. W. Cowgill, "Fluorescence and the structure of proteins II. Fluorescence of peptides containing tryptophan or tyrosine," Biochimica et Biophysica Acta, vol. 75, pp. 272-273, 1963.

[36] R. W. Cowgill, "Fluorescence and the structure of proteins. I. Effects of substituents on the fluorescence of indole and phenol compounds," Archives of Biochemistry and Biophysics, vol. 100, no. 1, pp. 36-44, 1963.

[37] J. Baselga and J. Albanell, "Mechanism of action of anti-HER2 monoclonal antibodies," Annals of Oncology, vol. 12, supplement 1, pp. S35-S41, 2001.

[38] J. Baselga, J. Albanell, M. A. Molina, and J. Arribas, "Mechanism of action of trastuzumab and scientific update," Seminars in Oncology, vol. 28, no. 5, supplement 16, pp. 4-11, 2001. 

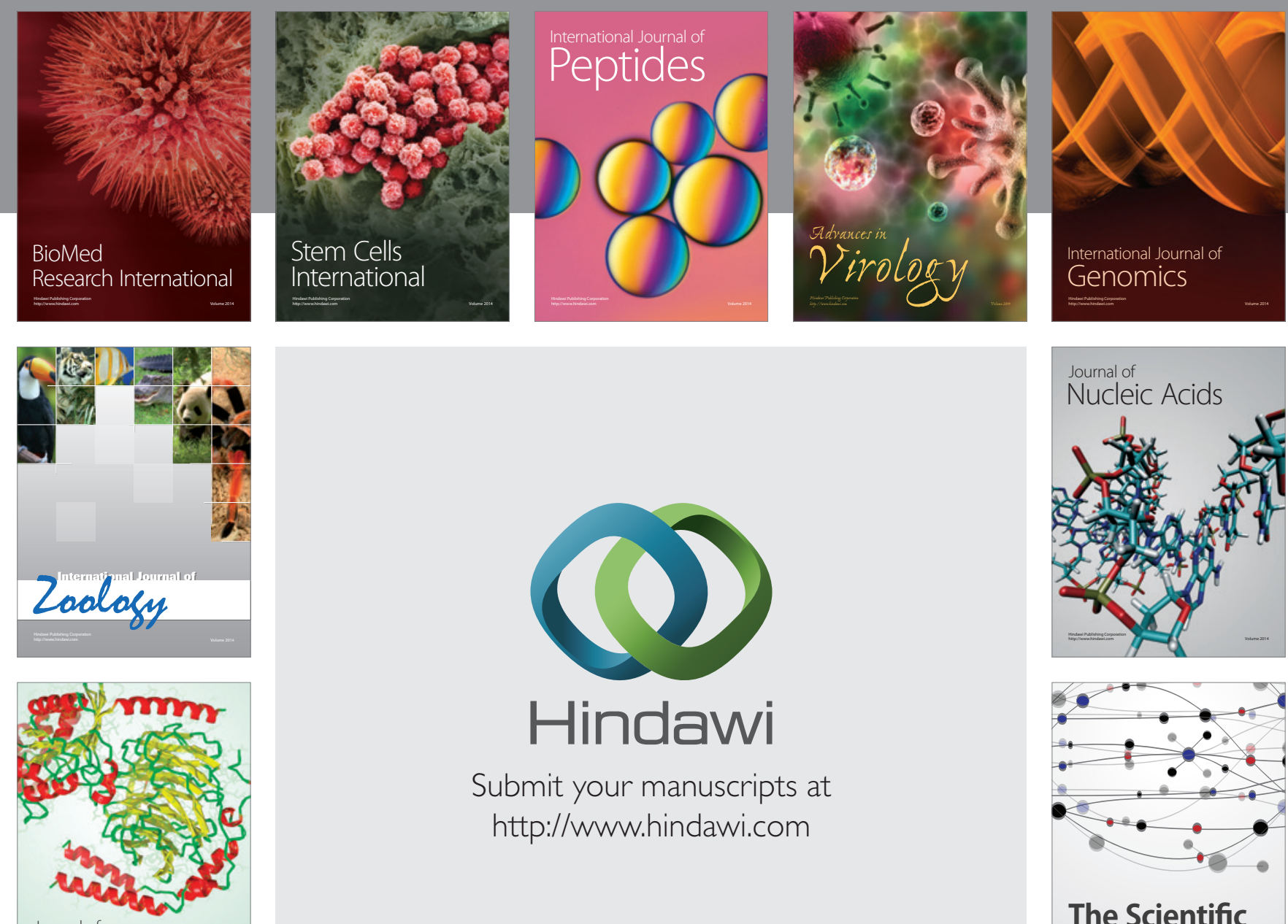

Submit your manuscripts at

http://www.hindawi.com

Journal of
Signal Transduction
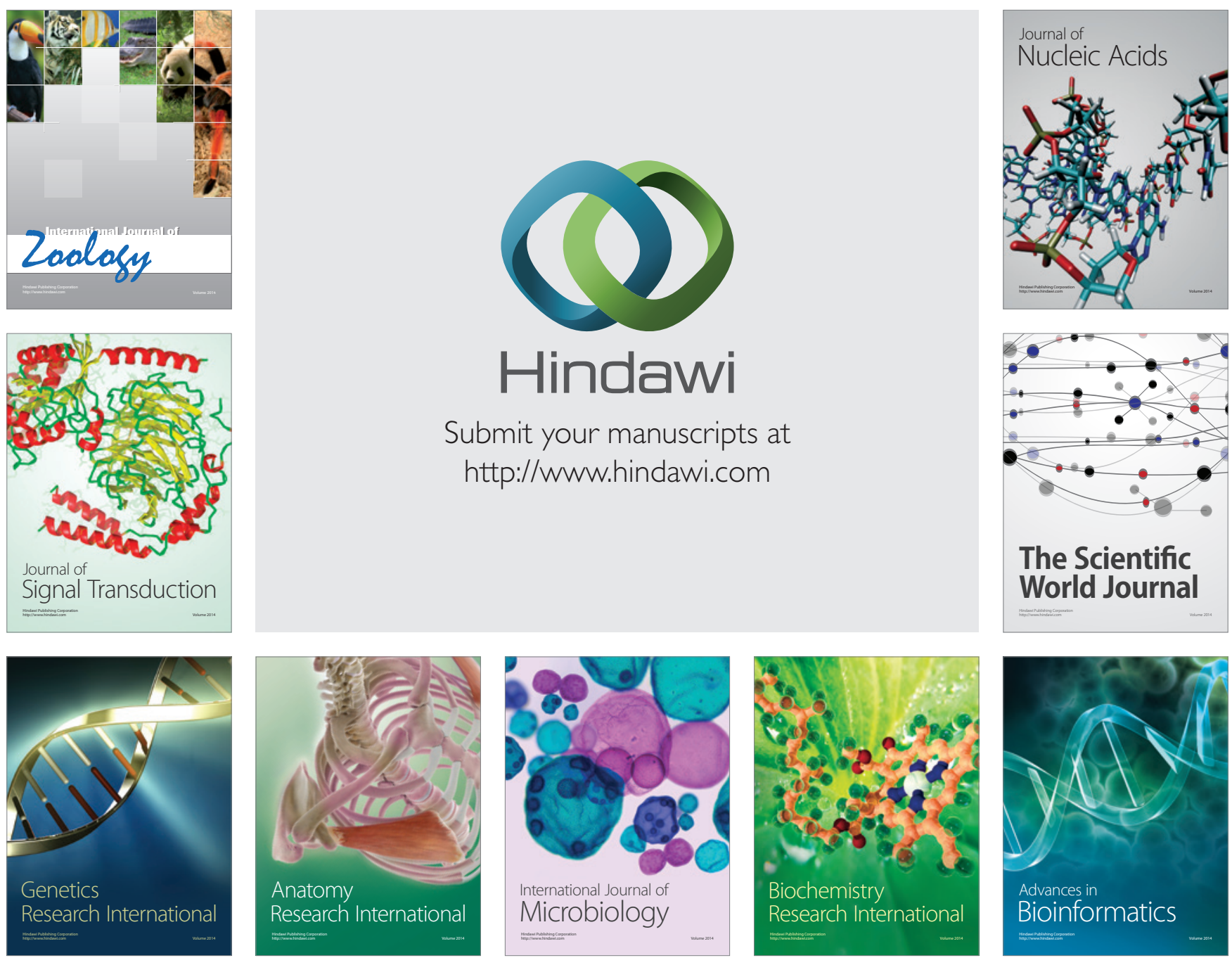

The Scientific World Journal
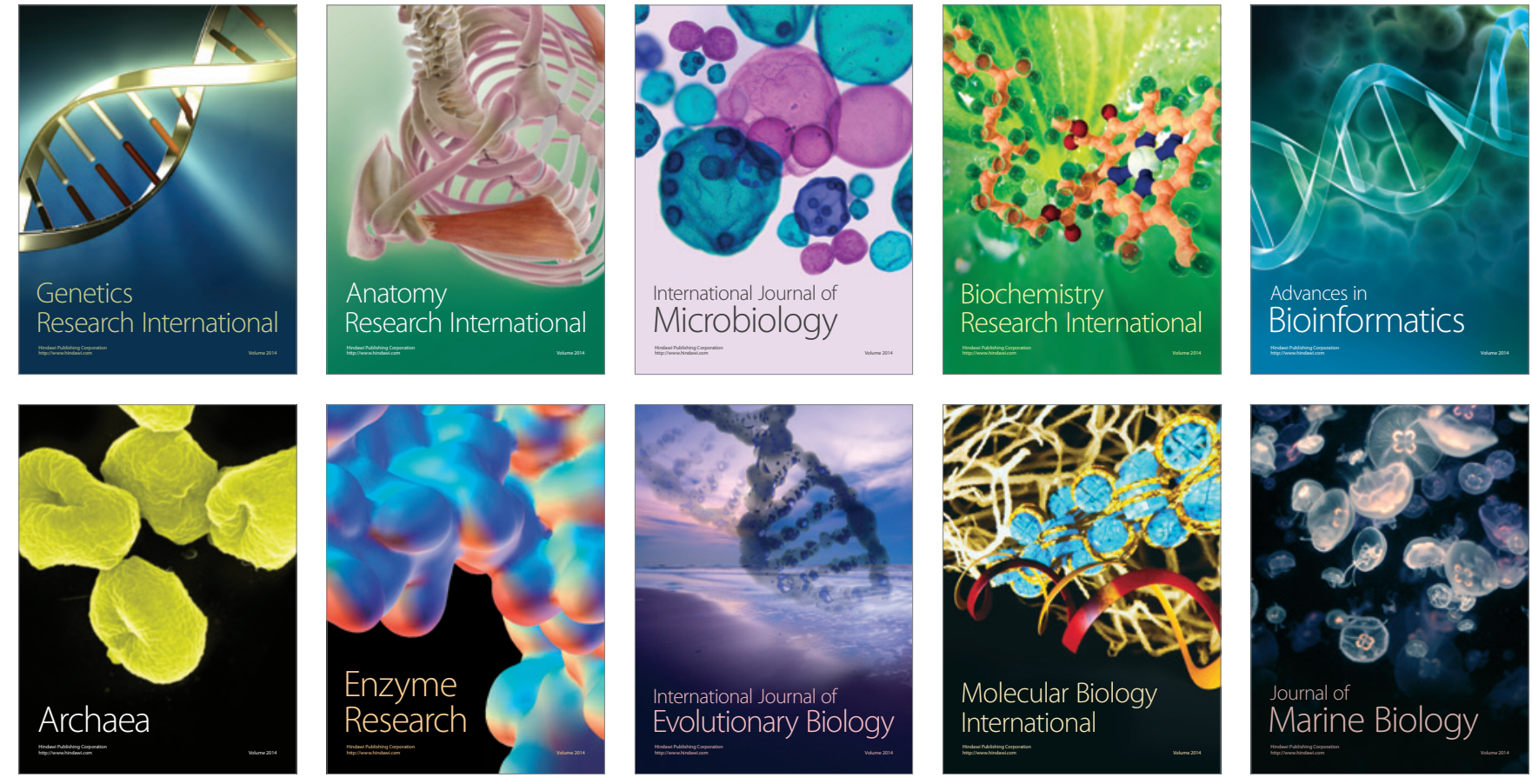